\title{
MEASURING SOIL MOISTURE WITH IMAGING RADARS
}

\author{
Pascale C. Dubois(*), Jakob van Zyl(*) and Ted Engman (**) \\ (*): Jet Propulsion Laboratory, California Institute of Technology \\ 4800 Oak Grove Drive, Pasadena CA 91109 \\ (**) NASA Goddard Space Flight Center \\ E-mail: pascale@blacks.jpl.nasa.gov Ph: (818) 354-0497
}

\section{INTRODUCTION}

An empirical model was developed to infer soil moisture and surface roughness from radar data. The accuracy of the inversion technique is assessed by comparing soil moisture obtained with the inversion technique to in situ measurements. The effect of vegetation on the inversion is studied and a method to eliminate the areas where vegetation impairs the algorithm is described.

\section{EXPERIMENTAL DATA AND MODEL DESCRIPTION}

Two sources of experimental data were used in the derivation of the empirical model. The University of Michigan's LCX POLARSCAT is a truck-mounted networkanalyzer-based scatterometer operating at three frequencies: $1.25,4.75$ and $9.5 \mathrm{GHz}$. The POLARSCAT data set (Oh et al., 1992) used in this study includes the co- and crosspolarized (hh, vv and hv) backscatters for four surfaces. The RASAM radar developed by the University of Bern, Switzerland is a truck-mounted radiometer-scatterometer with the scatterometer system operating at six frequencies between $2.5 \mathrm{GHz}$ and $11 \mathrm{GHz}$. A complete description of the instrument can be found in (Wegmuller, 1993). The RASAM data set includes measurements of the $h \mathrm{~h}, \mathrm{vv}, \mathrm{hv}$ and vh-polarized backscattering coefficients over a variety of surfaces.

Using these two data sets, the hh-polarized and vv-polarized backscattering coefficients $\sigma_{m}^{o}$ and $\sigma_{v v}^{o}$ were empirically found to follow these two equations:

$$
\begin{aligned}
& \sigma_{m h}^{0}=10^{-2.75} \frac{\cos \theta^{1.5}}{\sin \theta^{5}} 10^{0.28 \varepsilon_{\tan } \theta}(k h \sin \theta)^{1.4} \lambda^{0.7} \\
& \sigma_{v v}^{0}=10^{-2.35} \frac{\cos \theta^{3}}{\sin \theta^{3}} 10^{0.46 \varepsilon_{\tan } \theta}(k h \sin \theta)^{1.1} \lambda^{0.7}
\end{aligned}
$$

where $\theta$ is the incidence angle, $\varepsilon$ is the real part of the dielectric constant, $h$ is the RMS height of the surface, $k$ is the wave number and $\lambda$ is the wavelength in $\mathrm{cm}$.

The general backscatter behavior with roughness and dielectric constant is similar to the trend predicted by the small perturbation model and the physical optics model (Ulaby et al., 1986). The backscatters decrease with increasing incidence angle and with decreasing roughness. Restricting the validity of the model to $k h \leq 2.5$ and $\theta \geq 30^{\circ}$ will insure that the $\sigma_{m k}^{0} / \sigma_{v v}^{0}$ ratio is always less than 1 . We note that the roughness of the natural surfaces that the algorithm is applied to rarely exceeds $k h=2.5$ (corresponding for L-band to an RMS height of $10 \mathrm{~cm}$ ).

\section{EFFECTS OF VEGETATION AND CALIBRATION}

The inversion relies on the co-polarized channels and does not use the crosspolarized channels. This has several advantages. The first advantage is that the copolarized channels can be calibrated directly with passive targets like comer reflectors. 
The cross-polarized channel calibration is usually derived from measurements made on the co-polarized channels and as a result is less accurate than the calibration made on the co-polarized channels. The second advantage is the normally higher signal-to-noise ratio in the co-polarized channels than in the cross-polarized channels. Finally, the third advantage is that vegetation is known to have a relatively strong effect on the crosspolarized channel (Le Toan et al., 1992). It can therefore be expected that an inversion algorithm relying on the co-polarized channels will be more robust to the presence of vegetation than one relying on both the cross- and the co-polarized channels. Nevertheless, for a significant amount of vegetation, the algorithm will overestimate surface roughness and underestimate soil moisture.

Active microwave sensors have been shown to be good discriminators for biomass (Le Toan et al., 1992). This capability can be used to select the areas with low vegetation cover where the inversion can be applied. The L-band $\sigma_{h v}^{0} / \sigma_{v v}^{0}$ ratio image acquired over Chickasha, Oklahoma was compared to a SPOT-derived Normalized Difference Vegetation Index (NDVI) image (Tucker, 1979) over the same area. Overall, a pixel-to-pixel comparison between the two indices provides the regression curve. This curve shows that the L-band parameter is positively correlated to the NDVI. We found that masking out the areas for which the L-band $\sigma_{h v}^{0} / \sigma_{v v}^{0}$ ratio is greater than $-11 \mathrm{~dB}$, results in a reliable soil moisture estimate. The $-11 \mathrm{~dB} \sigma_{k v}^{0} / \sigma_{v v}^{0}$ ratio corresponds to NDVI of around 0.4 .

The study showed that a desired $4 \%$ accuracy in soil moisture estimate requires a $.5 \mathrm{~dB}$ accuracy of the relative calibration and $\mathrm{a} 2 \mathrm{~dB}$ accuracy in the absolute calibration. Both requirements are met by both the AIRSAR L-band and the SIR-C sensor. It should therefore be possible to derive accurate soil moisture maps for the data provided operationally by these sensors.

\section{SAR DATA INVERSION}

The inversion technique was first applied to Washita'92 AIRSAR data. The experiment followed a period of heavy rain so that the conditions on June 10, 1992 were very wet with standing water and saturated soils fairly common. No further rain fell during the next nine days and we were able to follow a drying pattern. The area covered in the AIRSAR Workshop Slide 1 is around $8 \mathrm{~km}$ by $10 \mathrm{~km}$. It was imaged by the AIRSAR system on six different days between June 10 and June 18, 1992. An L-band hh image is displayed at the lower right comer. The AG002 bare field which was extensively studied is outlined in the image. The other six images were obtained by inverting the $L$-band data acquired on the corresponding days. The dielectric constant maps were translated into soil moisture maps using the Hallikainen empirical curves (Hallikainen et al., 1985). The black areas in the soil moisture maps indicate areas where the vegetation cover is too thick for the inversion to be reliable. The scatter plot is a comparison between the in-situ measurements and the estimated values of soil moisture over the AG002 field. The RMS error of the soil moisture estimate is $1.6 \%$. The RMS height maps corresponding to the six data takes are very similar and indicate no trend in roughness changes. The quantitative results are presented in Table 1.

Washita'94 was an aircraft and shuttle experiment that occurred between April 9 and April 18 in which the shuttle took data on April 11 through 17 and the AIRSAR on April 10 and 11. Two of the sampled fields where data are already available are in the radar scene and meet the low vegetation criteria described earlier. The results from the inversion are included in Table 1 for both SIR-C and for AIRSAR.

The soil moisture inversion algorithm was also applied to an AIRSAR data set acquired over Spain in the summer of ' 91 during the EFEDA campaign (Bolle et al., 1992). Three of the sampled fields are in the radar scene and meet the low vegetation 
criteria described earlier. The results from the inversion are included in Table 1. Over all the areas where SAR data and ground truth measurements are available, the RMS error in soil moisture is $3.5 \%$ (see Table 1 and Figure 1).

\section{CONCLUSIONS}

We presented an empirical algorithm to infer soil moisture from imaging radar data over bare surfaces using two co-polarized radar cross-section measurements. The algorithm was developed with scatterometer data, and tested with several data sets acquired with the AIRSAR system; we also presented the first soil moisture images derived from spaceborne SIR-C SAR data. A comparison with in situ data shows that the algorithm infers soil moisture with an accuracy of better than $4 \%$. Best results are achieved when the surface roughness is such that $\mathrm{kh}<2.5(10 \mathrm{~cm}$ RMS height for Lband) and the incidence angle is larger than 30 degrees. We also quantified the calibration requirements of the algorithm and established that current operational multipolarization SAR systems such as AIRSAR and SIR-C routinely deliver images that meet or exceed these calibration requirements. We also presented evidence that the ratio of the cross-polarized return to the like-polarized return could be used to decide which areas the algorithm can be applied to and showed that this ratio was positively correlated with NDVI.

\begin{tabular}{|c|c|c|c|c|c|c|c|c|}
\hline Scene & Sensor & $\begin{array}{c}\text { Data } \\
\text { Take }\end{array}$ & Date & Field ID & $\begin{array}{c}\text { Mv } \\
\text { Msd } \\
{[\%]}\end{array}$ & $\begin{array}{c}\text { Mv } \\
\text { Est. } \\
{[\%]}\end{array}$ & $\begin{array}{c}\mathbf{h} \\
\text { Msd } \\
\text { (cm) }\end{array}$ & $\begin{array}{c}\mathbf{h} \\
\text { Est. } \\
\text { (cm) }\end{array}$ \\
\hline CHICKASHA & AIRSAR & 3902 & $6 / 10 / 92$ & AG002 & 28.7 & 29.2 & 1.19 & 1.18 \\
\hline CHICKASHA & AIRSAR & 3664 & $6 / 12 / 92$ & AG002 & 22.4 & 21.2 & 1.19 & 1.35 \\
\hline CHICKASHA & AIRSAR & 3359 & $6 / 13 / 92$ & AG002 & 24.1 & 23.5 & 1.19 & 1.09 \\
\hline CHICKASHA & AIRSAR & 3875 & $6 / 14 / 92$ & AG002 & 18.1 & 19.4 & 1.19 & 1.19 \\
\hline CHICKASHA & AIRSAR & 3883 & $6 / 16 / 92$ & AG002 & 13.6 & 17 & 1.19 & 1.24 \\
\hline CHICKASHA & AIRSAR & 3360 & $6 / 18 / 92$ & AG002 & 11.6 & 12.1 & 1.19 & 1.45 \\
\hline CHICKASHA & AIRSAR & 4040 & $6 / 17 / 92$ & AG002 & 17.5 & 18.5 & 1.19 & 1.45 \\
\hline CHICKASHA & AIRSAR & 4041 & $6 / 13 / 92$ & AG002 & 24.1 & 27.4 & 1.19 & 1.38 \\
\hline CHICKASHA & AIRSAR & Mosaic & $6 / 13 / 92$ & RG148 & 27.6 & 30.6 & & \\
\hline CHICKASHA & AIRSAR & Mosaic & $6 / 13 / 92$ & RG131 & 29.2 & 34.5 & & \\
\hline CHICKASHA & AIRSAR & 4254 & $4 / 12 / 94$ & Field 12 & 18.4 & 24.5 & & 1.58 \\
\hline CHICKASHA & AIRSAR & 4254 & $4 / 12 / 94$ & Field 15 & 24.8 & 21.8 & & 0.86 \\
\hline EFEDA & AIRSAR & 3146 & $6 / 13 / 90$ & Field 2 & 3.4 & 6.9 & 1.41 & 1.34 \\
\hline EFEDA & AIRSAR & 3146 & $6 / 13 / 90$ & Field 4 & 30.6 & 22.8 & 0.6 & 0.9 \\
\hline EFEDA & AIRSAR & 3146 & $6 / 13 / 90$ & Field 5 & 18.6 & 18.2 & 1.79 & 0.8 \\
\hline CHICKASHA & SIR-C & 10155 & $4 / 12 / 94$ & Field 12 & 18.4 & 20.2 & & 1.2 \\
\hline CHICKASHA & SIR-C & 10155 & $4 / 12 / 94$ & Field 15 & 24.8 & 30.3 & & 1.24 \\
\hline CHICKASHA & SIR-C & 10158 & $4 / 15 / 94$ & Field 12 & 9.9 & 12.5 & & 2 \\
\hline CHICKASHA & SIR-C & 10158 & $4 / 15 / 94$ & Field 15 & 12.5 & 11.8 & & 1.5 \\
\hline & & & & & & & & \\
\hline RMS ERROR & & & & & & 3.3 & & 0.34 \\
\hline
\end{tabular}

Table 1: Comparison between in situ measurements and estimated values. Mv stands for volumetric soil moisture, $\mathrm{h}$ for the RMS height. Est. and Msd indicate the radar derived values and the ground truth measurements. 


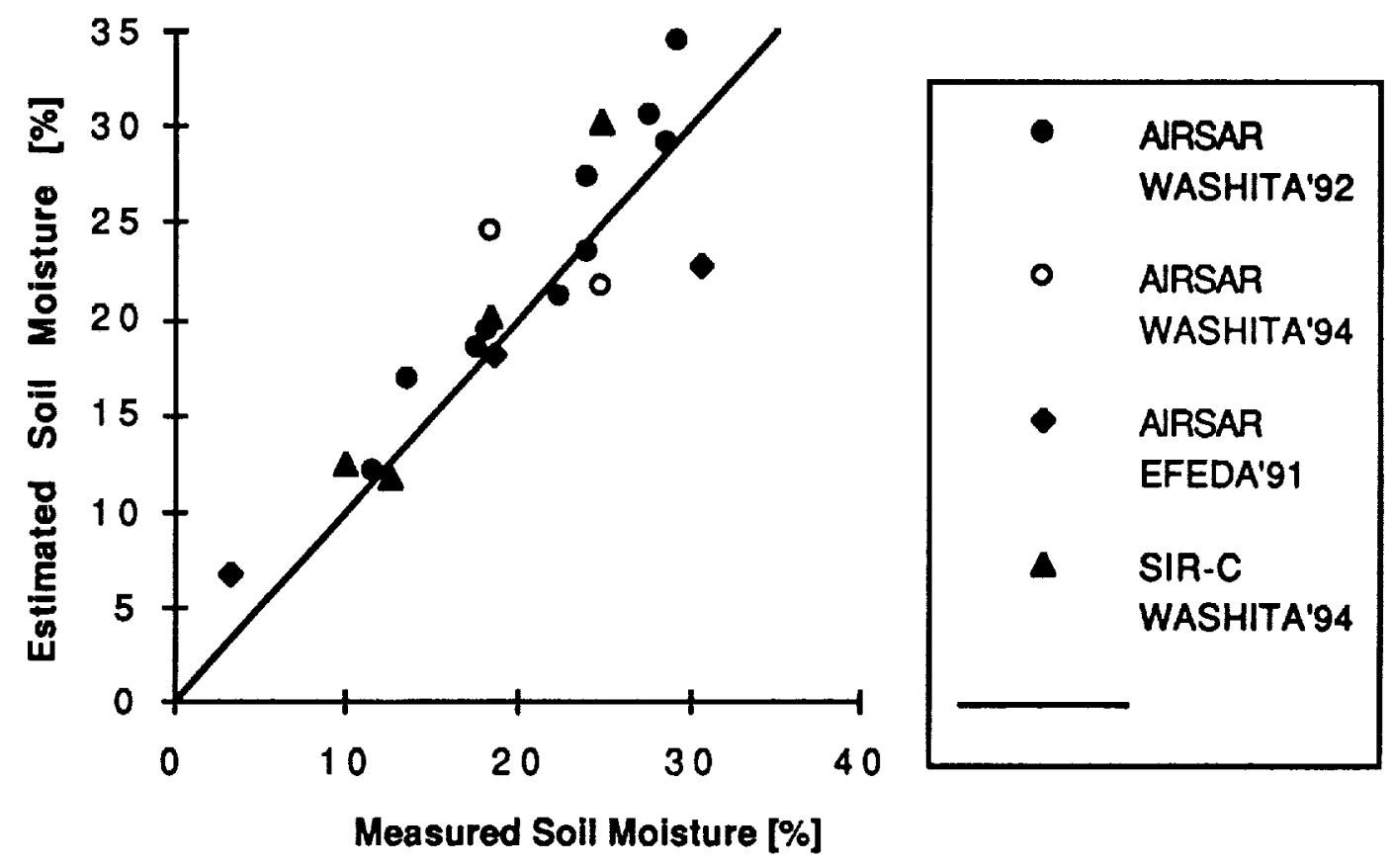

Figure 1: Radar derived soil moisture versus in situ measurements.

\section{ACKNOWLEDGMENT}

We would like to thank our colleagues from University of Michigan and University of Bern for sharing their scatterometer data and the corresponding ground truth with us. Our gratitude also goes to all the people involved in collecting the ground truth we used to validate this study. We would also like to acknowledge the help we received from Tom Jackson of the U.S. Deparment of Agriculture and his team. This work was performed at the Jet Propulsion Laboratory, California Institute of Technology under contract with the National Aeronautics and Space Administration.

\section{REFERENCES}

Bolle, H. J. and B. Streckenbach, 1992, " The ECHIVAL field experiment in a desertification-threatened area EFEDA, " First Annual Report to EC, published by the EFEDA-secretariat, Free University of Berlin, Berlin, Germany.

Hallikainen, M.T. , F. T. Ulaby, M. C. Dobson, M.A. El-Rayes and L. Wu, 1985, "Microwave dielectric behavior of wet soil - Part I: Empirical models and experimental observations," IEEE Trans. Geosc. Remote Sensing, vol. GE-23, pp. 25-34, 1985.

Oh, Y., K. Sarabandi and F. Ulaby, 1992, "An Empirical Model and an Inversion Technique for Radar Scattering from Bare Soil Surfaces," IEEE Trans. on GRS, vol. GE-30, No. 2.

Le Toan, T., A. Beaudoin and D. Guyon, 1992, "Relating forest biomass to SAR data," IEEE Transactions on Geoscience and Remote Sensing, Vol. 30, No. 2.

Tucker, C. J., 1979, "Red and photographic infrared combinations for monitoring vegetation," Remote Sensing of the Environment, vol. 8, pp. 127-150.

Ulaby, F.T., R. K. Moore, and A. K. Fung, 1986, Microwave Remote Sensing: Active and Passive, vol. 3, From theory to Applications, pp. 1065-2162, Dedham, MA: Artech House.

Wegmuller, U., 1993, "Active and Passive Microwave Signature Catalogue on Bare Soil (2-12 GHz), " Institute of Applied Physics, University of Bem, Switzerland, 1993. 